\title{
Nutritional Care for Valve Heart Disease Patients Under Warfarin Drug Treatment
}

\author{
Almojtaba AbdAlkhalig Ahmed Bakheet \\ Department of Family Sciences (Nutrition and Dietetics), Faculty of Education, University of Khartoum, Khartoum, Sudan
}

Emailaddress:

mugtabaahmed32@yahoo.com, moj76@uofk.edu

\section{To cite this article:}

Almojtaba AbdAlkhalig Ahmed Bakheet. Nutritional Care for Valve Heart Disease Patients Under Warfarin Drug Treatment. Science Research. Vol. 6, No. 1, 2018, pp. 24-28. doi: 10.11648/j.sr.20180601.14

Received: December 11, 2017; Accepted: January 30, 2018; Published: February 27, 2018

\begin{abstract}
This review gives a survey on the representative role concerning the nutritional care of valve heart disease patients under warfarin drug treatment, the objectives of this work is to give a nutritional views to the valve heart disease from the disease definition, determining Risks, Complications, surgical outcome and risk of procedure, It also highlights the blood coagulation, warfarin drug and its effects and the role of nutritional support in the treatment of valve heart disease patients.
\end{abstract}

Keywords: Valve Heart Disease, Warfarin Drug and Its Effects, Nutritional Support

\section{Introduction}

Valve heart disease is any disease process involving one or more of the valves of the heart, the aortic and mitral valves on the left and the pulmonary and tricuspid valves on the right. A heart valve normally allows blood to flow in only one direction through the heart. The four valves commonly represented in a mammalian heart determining the pathway of blood flow through the heart. A heart valve opens or closes incumbent on differential blood pressure on each side [1].

The four main valves in the heart are:

1. The two atrioventricular (AV) valves, the mitral valve (bicuspid valve), and the tricuspid valves, which are between the upper chambers (atria) and the lower chambers (ventricles).

2. The two semilunar (SL) valves, the aortic valve and the pulmonary valve, which are in the arteries leaving the heart.

The mitral valve and the aortic valve are in the left heart; the tricuspid valve and the pulmonary valve are in the right heart.

Valve problems may be congenital inborn or acquired (due to another cause later in life). Treatment may be with medication but often depending on the severity involves valve repair or replacement insertion of an artificial heart valve. Specific situations include those where additional demands are made on the circulation, such as in pregnancy [2].

The most common types of pulmonary valve diseases are: pulmonary valve stenosis, pulmonary valve insufficiency, pulmonary valve incompetence and pulmonary valve regurgitation.

The International statistical classification of diseases classifies non rheumatic pulmonary valve diseases as I37. Both tricuspid and pulmonary valve diseases are less common than aortic or mitral valve diseases due to the lower pressure those valves experience. [3].

Heart valve dysplasia is an error in the development of any of the heart valves, and a common cause of congenital heart defect in humans as well as animals; tetralogy of fallout is a congenital heart defect with four abnormalities, one of which is stenosis of the pulmonary valve. Epstein's anomaly is an abnormality of the tricuspid valve [4].

Valvular heart disease resulting from rheumatic fever is referred to as "rheumatic heart disease". While developed countries once had a significant burden of rheumatic fever and rheumatic heart disease, medical advances and improved social conditions have dramatically reduced their incidence. Many developing countries, as well as indigenous populations within developed countries, still carry a significant burden of rheumatic fever and rheumatic heart disease and there has been a resurgence in efforts to eradicate the diseases in these populations. Inflammation of the heart valves due to any cause is called endocarditis; this is usually due to bacterial infection but may also be due to cancer, certain autoimmune conditions and hypereosinophilic syndrome. Certain medications have been associated with valvular heart disease, most prominently 
ergotamine derivatives pergolide and cabergoline [3].

The evaluation of individuals with valvular heart disease who are or wish to become pregnant is a difficult issue. Issues that have to be addressed include the risks during pregnancy to the mother and the developing fetus. Normal physiological changes during pregnancy require, on average, a 50\% increase in circulating blood volume that is accompanied by an increase in cardiac output that usually peaks between the mid-portion of the second and third trimesters. The increased cardiac output is due to an increase in the stroke volume, and a small increase in heart rate, averaging 10 to 20 beats per minute. Additionally uterine circulation and endogenous hormones can cause systemic vascular resistance to decrease and a disproportionately lowering of diastolic blood pressure which causes a wide pulse pressure. Inferior vena caval obstruction from a gravid uterus in the supine position can result in an abrupt decrease in cardiac preload, which leads to hypotension with weakness and lightheadedness. During labor and delivery cardiac output increases more in part due to the associated anxiety and pain, as well as due to uterine contractions which will cause an increasing in systolic and diastolic blood pressure.

\section{Determining Risks}

Patients who may be indicated for heart valve replacement should discuss their risks of surgery with both their cardiologist and cardiothoracic surgeon. Considerations that should be taken into account include age, overall health, other medical conditions and heart function. While no predictions are absolute, patients should be made aware of potential complications that can occur following the surgery.

\section{Minor Complications}

Surgical complications as a result of valve replacement fall into two categories: minor, which are typically temporary conditions that subside in a matter of weeks, and major, which require surgery or medical attention.

Minor complications can be due to reactions from general anesthesia, including nausea and vomiting. Other complications include skin numbness, bleeding from the surgical site, bruising or even an abnormal heartbeat as the heart becomes accustomed to the new valve.

\section{Major Complications: Heart Attack}

The risk of complications builds if a patient has chronic conditions, such as heart disease, diabetes, lung disease or kidney disease. One of the complications includes heart attack, which occurs in four out of every 1,000 procedures, according to EMedTV.com.

The heart must work harder to pump blood and oxygen throughout the body during surgery. If a vessel or artery (or even multiple ones) are blocked, this can cause the heart to pump insufficient oxygen to the heart muscle. As a result, a heart attack can occur.

\subsection{Stroke}

A complication that can occur in 4 out of every 100 valve replacement procedures, a stroke can occur when a blood clot breaks off in the heart's arteries. This clot can then travels to the brain and block arteries, which causes a stroke. A stroke is considered a serious complication because the longer a person's brain is deprived of oxygen, the more likely a person is to experience paralysis, speech problems or even death.

\subsection{Organ Malfunction}

In addition to damage to the heart, risks associated with valve replacement include damage to the lungs, kidneys or other surrounding organs. Because the heart plays a role in the function of every part of the body by supplying blood and oxygen, heart valve replacement may cause unexpected damage to other organs. If damage to the kidneys occurs, dialysis (which uses a machine to mimic kidney function) may be necessary.

\section{Surgical Outcome and Risk of Procedure}

The risk of death or serious complications from aortic valve replacement is typically quoted as being between 1-3\%, depending on the health and age of the patient, as well as the skill of the surgeon. Older patients, as well as those who are frail and/or have multiple co-morbidities may face significantly higher surgical risk [5].

Minimally invasive cardiac surgery. More recently, some cardiac surgeons have been performing aortic valve replacement procedures using an approach referred to as minimally invasive cardiac surgery (MICS), in which the surgeon replaces the valve through small incisions between two to four inches in length using specialized surgical instruments rather than by cutting a six to ten-inch incision down the center of the sternum.

Hospital stay and recovery time after valve replacement is differ, but the patient will frequently stay in an intensive care unit for 12-36 hours. The patient is often able to go home after this, in about four days, unless complications arise. Common complications include heart block, which typically requires the permanent insertion of a cardiac pacemaker. Recovery from valve replacement will take about three months, if the patient is in good health. Patients are advised not to do any heavy lifting for 4-6 months after surgery, to avoid damage to the sternum (the breast bone) [5].

\section{Blood Coagulation}

Blood coagulation is one of the mechanisms of (blood homeostasis prevention of blood loss) and comprises a complicated interaction of multiple enzymes and proteins [6]. The clot begins to develop in 15 to 20 seconds if the trauma to the vascular wall has been severe and in 1 to 2 minutes if the trauma has been minor. Within 3 to 6 minutes after rupture of 
a vessel, if the vessel opening is not too large, the entire opening or broken end of the vessel is filled with clot. After 20 minutes to an hour, the clot retracts; this closes the vessel still further. Platelets also play important role in this clot retraction [7].Anticoagulants for clinical use. In some thromboembolic conditions it is desirable to delay the coagulation process. Various anticoagulants have been developed for this purpose. The ones most useful clinically are heparin and the cumarins.

Oral anticoagulants are used to create a partial deficiency of the active form of vitamin $\mathrm{K}$, which is responsible for maintaining normal blood coagulation. By inhibiting the action of vitamin $\mathrm{K}$, there is a reduced risk of abnormal blood clotting.

Indications of oral anticoagulants are typically prescribed for 3 to 6 months to treat the following conditions:

venous thrombosis

pulmonary embolism

myocardial infarction

Persons with prosthetic heart valves, atrial fibrillation with embolization, or heredity disorders that result in a hypercoagulant state may be treated with anticoagulants indefinitely [8].

\section{Warfarin}

It is also known under the brand names (Coumadin, Jantoven, Marevan, Lawarin, Waran, and Warfarin) is an anticoagulant normally used in the prevention of thrombosis and thromboembolism, the formation of blood clots in the blood vessels and their migration elsewhere in the body respectively. It was initially introduced in 1948 as a pesticide against rats and mice and is still used for this purpose, although more potent poisons such as brodifacoum have since been developed. In the early 1950s warfarin was found to be effective and relatively safe for preventing thrombosis and embolism (abnormal formation and migration of blood clots) in many disorders. It was approved for use as a medication in 1954 and has remained popular ever since; warfarin is the most widely prescribed oral anticoagulant drug in north America.

\subsection{Medical Uses}

Warfarin is used to decrease the tendency for thrombosis or as secondary prophylaxis (prevention of further episodes) in those individuals that have already formed a blood clot (thrombus). Warfarin treatment can help prevent formation of future blood clots and help reduce the risk of embolism (migration of a thrombus to a spot where it blocks blood supply to a vital organ)

Warfarin is best suited for anticoagulation (clot formation inhibition) in areas of slowly running blood (such as in veins and the pooled blood behind artificial and natural valves) and in blood pooled in dysfunctional cardiac atria. Thus, common clinical indications for warfarin use are a trial fibrillation, the presence of artificial heart valves, deep venous thrombosis, and pulmonary embolism (where the embolized clots first form in veins). Warfarin is also used in anti-phospholipid syndrome. It has been used occasionally after heart attacks (myocardial infarctions), but is far less effective at preventing new thromboses in coronary arteries. Prevention of clotting in arteries is usually undertaken with antiplatelet drugs, which are act by a different mechanism from warfarin (which normally has no effect on platelet function).

\subsection{Mechanism of Action}

In the normal coagulation cascade, factors II, VII, IX, and X undergo $\gamma$-glutamyl-carboxylation to become functional. The enzyme catalyzing this reaction, $\gamma$-glutamyl-carboxylase, requires reduced vitamin $\mathrm{K}$ hydroquinone (VitKH2) as a source of protons to complete carboxylation. For this reason, factors II, VII, IX, and $\mathrm{X}$ are referred to as the vitamin $\mathrm{K}$ - dependent coagulation factors. Vitamin $\mathrm{K}$ epoxide is a by product of $\gamma$-carboxylation. The enzyme vitamin Kepoxide reductase (VKOR) recycles vitamin K epoxide to VitKH2 to sustain formation of coagulation factors that can be activated in the maintenance of the coagulation process. Warfarin is a potent inhibitor of VKOR. Anticoagulation occurs when warfarin inhibits VKOR, decreasing the amount of VitKH2 available to sustain functional vitamin K-dependent coagulation factors [9].

Carboxylation requires the reduced form of vitamin $\mathrm{K}$ (vitamin KH2). Coumarins block the formation of vitamin $\mathrm{KH} 2$ by inhibiting the enzyme 16 vitamin K epoxide reductase, thereby limiting the $\gamma$-carboxylation of the vitamin $\mathrm{K}$ dependent coagulant proteins. In addition, the vitamin $\mathrm{K}$ antagonists inhibit carboxylation of the regulatory anticoagulant proteins $\mathrm{C}$ and $\mathrm{S}$ [10].Anticoagulation occurs when warfarin inhibits VKOR, decreasing the amount of vitamin $\mathrm{KH} 2$ available to sustain functional vitamin $\mathrm{K}$ dependent coagulation factor.

\subsection{Adverse Effect}

\subsubsection{Hemorrhage}

The only common side effect of warfarin is hemorrhage (bleeding). The risk of severe bleeding is small but definite (a median annual rate of 0.9 to $2.7 \%$ has been reported and any benefit needs to outweigh this risk when warfarin is considered as a therapeutic measure. Risk of bleeding is augmented if the INR is out of range (due to accidental or deliberate overdose or due to interactions), and may cause hemoptysis (coughing up blood), excessive bruising, bleeding from nose or gums, or blood in urine or stool.

The risk of bleeding is increased when warfarin is combined with antiplatelet drugs such as clopidogrel, aspirin, or other nonsteroidal anti-inflammatory drugs. The risk may also be increased in elderly patients and in patients on hemodialysis.

\subsubsection{Pregnancy}

Warfarin is contraindicated in pregnancy, as it passes through the placental barrier and may cause bleeding in the fetus; warfarin use during pregnancy is commonly associated with spontaneous abortion, stillbirth, neonatal death, and preterm birth. Coumarins (such as warfarin) are also 
teratogens, that is, they cause birth defects; the incidence of birth defects in infants exposed to warfarin in utero appears to be around $5 \%$, although higher figures (up to $30 \%$ ) have been reported in some studies. Depending on when exposure occurs during pregnancy, two distinct combinations of congenital abnormalities can arise.

Usually, warfarin is avoided in the first trimester, and a low molecular weight heparin such as enoxaparin is substituted. With heparin, risk of maternal hemorrhage and other complications is still increased, but heparins do not cross the placental barrier and therefore do not cause birth defects. Various solutions exist for the time around delivery.

\subsection{International Normalizing Ratio (INR) Test}

There is a blood test used to measure the time it takes for blood to clot, it is referred to pro time (PT). The PT is reported as the(INR). The INR is a standardized way of expressing the PT value. The INR ensures that PT results obtained by different laboratories can be compared. It is important to monitor the INR (at least once a month and sometimes as often as twice weekly) to make sure that the level of warfarin remains in the effective range. If the INR is too low, blood clots will not be prevented, but if the INR is too high, there is an increased risk of bleeding. For patients with bioprosthetic valves, warfarin therapy with a target INR of 2.5 (range, 2.0 to 3.0) is recommended for valves in the mitral position and is suggested for valves in the aortic position for the first 3 months after valve insertion [11].

\section{Nutritional Care}

\subsection{Food Interactions}

Understanding how diet can affect in INR level is an important aspect of living a healthy life, but in valve heart disease patients under warfarin treatment is more important . Regardless of vitamin $\mathrm{K}$ content, all foods have the potential to interact and reduce the effectiveness of warfarin, so it is important to pay close attention to the foods that the patients take.

The oral anticoagulant dose should be established based on the patient's normal vitamin $\mathrm{K}$ intake. After the dose is established, a reasonable goal is to maintain within $\quad 250 \mu \mathrm{g}$ of the vitamin $\mathrm{K}$ baseline. If major changes in food intake occur, the anticoagulant level may need to be reestablished. The most frequent reason for an increase in vitamin $\mathrm{K}$ is when a patient starts a weight-reduction diet and includes a greater number of vegetables that are high in vitamin $\mathrm{K}$. Other reasons may include an adjustment in diet because of hospitalization or a change in seasons.

Up until 1984, the literature contained case reports involving the intake of enteral nutrition support products by patients taking anticoagulants. Since that time, enteral nutrition products have been reformulated with lower contents of vitamin $\mathrm{K}$; subsequently there has been a decrease in the number of case reports with changes in coagulation status[8]. However, it has been cited that anticoagulant absorption may be impaired as a result of physical or chemical interactions with other ingredients contained in newly formulated enteral feeding preparations. In these cases, it is recommended to withhold the enteral feeding 3 hours before or after the drug administration [12].

Drug-drug interactions that increase or decrease the effect should be evaluated before concluding that diet is responsible for change in anticoagulant response. Drugs that increase the effect are agents for gout, anabolic steroids, antiarrhythmic agents, antibiotics, antifungal agents, antihyperlipidemic agents, cimetidine, disulfiram, isoniazid, omeprazole, sulfonylureas, and tamoxifen citrate. Drugs that decrease the effect are alcohol, anticonvulsant agents, cholestyramine, griseofulvin, oral contraceptives, rifampin, sucralfate, and vitamin $\mathrm{K}$ [13].

It is important to remain consistent with beverages intake in order to avoid fluctuations in INR level. More frequent INR testing is recommended to help identify any food and drug interactions with warfarin drug [14].

\subsection{Dietary Pattern}

Changes in daily vitamin $\mathrm{K}$ intake may contribute to marked variations in the International Normalized Ratio (INR) coagulation index in patients receiving oral warfarin anticoagulant therapy, with potentially serious adverse outcomes. Thus, patients receiving warfarin therapy are routinely counseled regarding this drug-nutrient interaction and are instructed to maintain consistent vitamin $\mathrm{K}$ intakes [15].

It would be useful to estimate the vitamin $\mathrm{K}$ content of a patient's diet in certain situations. These include at the initiation of oral anticoagulant therapy, before a requested change in the patient's dietary pattern, and when the prothrombin time (PT) changes and cannot be explained by a drug-drug interaction or change in disease status.

The Dietary Reference Intake (DRI) for vitamin K is $1 \mu \mathrm{g}$ $/ \mathrm{kg}$ of body weight per day. The actual intake of vitamin $\mathrm{K}$ in Western countries is estimated to be 300 to $500 \propto \mathrm{g}$ daily, but actual intake depends on consumption of foods high in vitamin K. Unlike other fat-soluble vitamins, stores of vitamin $\mathrm{K}$ are rapidly depleted if intake is deficient [8]. This information may be helpful in assessing the vitamin $\mathrm{K}$ level of a patient who has had a low intake of food for a week or longer.

Patients should be educated on dietary changes that have an impact on anticoagulant therapy. They also should be informed of foods that are high in vitamin $\mathrm{K}$ (eg, cauliflower, broccoli, cabbage, spinach, kale, other dark green leafy vegetables, liver, soybeans, and green tea). They should be encouraged to keep their diet consistent with their present pattern. However, if there is a change in diet that includes these foods, they should contact their physician and have their PT monitored.

It is best for the patient taking anticoagulants to abstain from alcohol. However, an occasional light drink does not appear to be detrimental to the coagulation status [8]. 


\subsection{Botanical Effect}

Herbs caution should be exercised when botanical medicines are taken concomitantly with coumadin. Few adequate, well-controlled studies exist evaluating the potential for metabolic and/or pharmacologic interactions between botanicals and coumadin. Due to a lack of manufacturing standardization with botanical medicinal preparations, the amount of active ingredients may vary. This could further confound the ability to assess potential interactions and effects on anticoagulation. It is good practice to monitor the patient's response with additional PT/INR determinations when initiating or discontinuing botanicals [4] Specific botanicals reported to affect warfarin therapy include garlic ginseng, and cranberry products are associated most often with an increase in the effects of warfain. Some botanicals may cause bleeding events when taken alone (e.g. garlic) and may have anticoagulant, antiplatelet, and/or fibrinolytic properties. These effects would be expected to be additive to the anticoagulant effects of warfarin. Conversely, other botanicals may have coagulant properties when taken alone or it may decreases the effects of warfarin [16].

\section{Conclusion}

The applications of the nutritional care of valve heart disease patients, from the fundamental Approaches of the disease definition, determining Risks, Complications and surgical outcome and risk of procedure, It also it highlighted the Blood coagulation process, Warfarin drug and its effects on treatment of these patients,.

Once the benefits of the role of nutritional care in the treatment of valve heart disease patients which will envisaged in the near future studies, so scientists with an interest in nutritional care may also find that this work provide exciting opportunities.

\section{References}

[1] American Heart Association, Inc -- 10000056 Heart and Stroke Encyclopedia. American Heart Association, Inc. Retrieved 2010-08-05.

[2] Jr S S, Allen J, Blair S N, et al. AHA/ACC guidelines for secondary prevention for patients with coronary and other atherosclerotic vascular disease: 2006 update: endorsed by the National Heart, Lung, and Blood Institute.[J]. Journal of the American College of Cardiology, 2006, 47(10): 2130-9.
[3] Vukasinović M, Djukić V, Stanković P, et al. International Statistical Classification of Diseases and Related Health Problems [J]. Acta Chirurgica Iugoslavica, 2009, 56 (3): 65-9.

[4] Andersohn F, Suissa S, Garbe E. Cyclooxygenase inhibitors and cardiovascular risk [J]. Jama the Journal of the American Medical Association, 2007, 297 (6): 587-8.

[5] Torracca L, Schreuder J J, Quarti A, et al. Acute effects of beating heart coronary surgery on left ventricular performance. [J]. Annals of Thoracic Surgery, 2002, 74(4): 1348-1352.

[6] Wajima T, Isbister G K, Duffull S B. A comprehensive model for the humoral coagulation network in humans. [J]. Clinical Pharmacology \& Therapeutics, 2009, 86 (3): 290-298.

[7] Guyton, Arthur C. Guyton and Hall textbook of medical physiology/[M]. Saunders/Elsevier, 2011.

[8] Harris J. Interaction of dietary factors with oral anticoagulants: review and applications. J Am Diet Assoc. 1995; 95: 580-584.

[9] Calonge N, Petitti D B, Dewitt T G, et al. Aspirin for the prevention of cardiovascular disease: U.S. Preventive Services Task Force recommendation statement.[J]. Annals of Internal Medicine, 2009, 150 (6): 396.

[10] Hirsh J, Fuster V, Ansell J, et al. American Heart Association/American College of Cardiology Foundation guide to warfarin therapy. [J]. Journal of the American College of Cardiology, 2003, 107(12): 1692.

[11] Goldhaber S Z. Optimal duration of anticoagulation after venous thromboembolism: fixed and evidence-based, or flexible and personalized? [J]. Annals of Internal Medicine, 2009, 150(9): 644-646.

[12] Yamreudeewong W, Henann N, Fazio A, Lower D, Cassidy T. Drug-food interactions in clinical practice. J Fam Pract. 1995; 40(4): $376-384$

[13] Brigden M. Oral anticoagulant therapy. Postgrad Med 1996; 99(6): 81-100.

[14] Girolami A, Scandellari R, Zanon E, et al. Non-catheter associated venous thrombosis in hemophilia A and B. A critical review of all reported cases. [J]. Journal of Thrombosis \& Thrombolysis, 2006, 21(3): 279-84.

[15] Couris R, Tataronis G, Mccloskey W, et al. Dietary vitamin K variability affects International Normalized Ratio (INR) coagulation indices[J]. International journal for vitamin and nutrition research. Internationale Zeitschriftfür Vitamin- und Ernährungsforschung. Journal international de vitaminologieet de nutrition, 2006, 76(2): 65.

[16] Izzo A A, Carlo G D, Borrelli F, et al. Cardiovascular pharmacotherapy and herbal medicines: the risk of drug interaction[J]. International Journal of Cardiology, 2005, 98(1): 1. 